\title{
THE COMPLEX INVERSION FORMULA REVISITED
}

\author{
MARKUS HAASE \\ (Received 3 October 2006; revised 25 June 2007) \\ Communicated by A. J. Pryde
}

\begin{abstract}
We give a simplified proof of the complex inversion formula for semigroups and, more generally, solution families for scalar-type Volterra equations, including the stronger versions on unconditional martingale differences (UMD) spaces. Our approach is based on (elementary) Fourier analysis.

2000 Mathematics subject classification: 34G10, 43A50, 44A10, 47A60, 47D03, 47D06, 47D09.

Keywords and phrases: $C_{0}$ semigroup, integrated semigroup, UMD space, complex inversion, Laplace transform, Volterra equation.
\end{abstract}

\section{Introduction}

In this paper we are concerned with the following question. Let $X, Y$ be Banach spaces and let $S:[0, \infty) \longrightarrow \mathcal{L}(X, Y)$ be a strongly continuous mapping of finite exponential type $\omega_{0}(S)$. In what sense and under what conditions does the complex inversion inversion formula

$$
\lim _{N \rightarrow \infty} \frac{1}{2 \pi i} \int_{\omega-i N}^{\omega+i N} e^{t z}(\mathcal{L} S)(z) d z=S(t) \quad(t>0),
$$

hold true? (Here $\omega>\omega_{0}(S)$ is fixed and $\mathcal{L} S$ denotes the Laplace transform of $S$.)

Actually we are interested in the case that $S$ is a solution family to a scalar-type Volterra equation (see Section 4), in particular that $S$ is a $C_{0}$ semigroup. However, as in [1, Theorem 2.3.4] we do not confine ourselves to these applications and start very generally.

Theorem 2.3.4 from [1] states that (1.1) holds in an 'integrated form'. From this one can then derive the standard result on semigroups (strong convergence on the domain of the generator). Driouich and El-Mennaoui [4] showed that in case that $X$ has the

(C) 2008 Australian Mathematical Society 1446-7887/08 \$A2.00 + 0.00 
unconditional martingale differences (UMD) property, the convergence is strong on all of $X$. This was subsequently generalized from semigroups to solution families for scalar-type Volterra equations by Cioranescu and Lizama in [3].

The aim of the present paper is to present new and much shorter proofs of these results, eventually even generalizing them. Our approach uses some elementary Fourier analysis and has the advantage that the recent 'UMD results' become at least as simple as the classical results, if not simpler. The results obtained are also more specific with respect to what happens with the approximation for small times (compare, for example, Theorem 3.5 here with [5, Corollary III.5.15]).

All of our results on the complex inversion formula remain true when we let the lower and the upper bound of the integral in (1.1) tend to infinity independently. One has to replace the Dirichlet kernel in our discussion with a somewhat more complicated expression, but the proofs are essentially the same.

1.1. Preliminary remarks and definitions Here and in the following $X, Y, Z$ always denote complex Banach spaces. The symbol $\mathbf{1}$ is used to denote the characteristic function of the positive real axis, that is $\mathbf{1}=\chi_{[0, \infty)}$. So $\mathbf{1}^{\prime}=\delta_{0}$ in the distributional sense, where $\delta_{0}$ is the Dirac measure at 0 . We write simply $t$ to denote the real coordinate $(t \longmapsto t)$. All functions that live on $[0, \infty)$ are tacitly extended to $\mathbb{R}$ by 0 on $(-\infty, 0)$. For a mapping $S:[0, \infty) \longrightarrow \mathcal{L}(X, Y)$ and $\omega \in \mathbb{R}$ we define its exponential shift $S_{\omega}$ by

$$
S_{\omega}(t):=e^{-\omega t} S(t) \quad(t \geq 0) .
$$

The exponential type of $S$ is

$$
\omega_{0}(S):=\inf \left\{\omega \in \mathbb{R} \mid \exists M \geq 0:\|S(t)\| \leq M e^{\omega t}(t \geq 0)\right\} .
$$

If $S$ is strongly measurable and of finite exponential type, we denote by

$$
(\mathcal{L} S)(z):=\operatorname{strong}-\int_{0}^{\infty} e^{-z t} S(t) d t \quad\left(\operatorname{Re} z>\omega_{0}(S)\right)
$$

its Laplace transform. If $S:[0, \infty) \longrightarrow \mathcal{L}(X, Y)$ and $T:[0, \infty) \longrightarrow \mathcal{L}(Y, Z)$ are both strongly measurable and of finite exponential type, then the convolution $S * T$ : $[0, \infty) \longrightarrow \mathcal{L}(X, Y)$ given by

$$
(T * S)(t) x:=\int_{0}^{\infty} T(t-s) S(s) x d s \quad(x \in X),
$$

is well-defined, strongly continuous and of finite exponential type; furthermore, one has

$$
(T * S)_{\omega}=T_{\omega} * S_{\omega} \quad \text { and } \quad \mathcal{L}(T * S)=(\mathcal{L} T)(\mathcal{L} S) .
$$

In addition to this type of convolution we encounter (in Section 4) $\mu * S$, where $S:[0, \infty) \longrightarrow \mathcal{L}(X, Y)$ is strongly continuous and $\mu$ is a locally finite complex Borel measure on $[0, \infty)$. The convolution $\mu * S:[0, \infty) \longrightarrow \mathcal{L}(X, Y)$ is then given as

$$
(\mu * S)(t) x:=\int_{0}^{t} S(t-s) x \mu(d s) \quad(x \in X),
$$


and is again strongly continuous. With the obvious definition of $\mu_{\omega}$ we have $(\mu * S)_{\omega}=\mu_{\omega} * S_{\omega}$; if $\mu_{\omega}$ happens to be a bounded measure, $\mathcal{L} \mu$ is defined in the obvious way, and one has $\mathcal{L}(\mu * S)=(\mathcal{L} \mu)(\mathcal{L} S)$.

A third situation involves functions on the whole real line, and is described in the following. A strongly measurable mapping $S: \mathbb{R} \longrightarrow \mathcal{L}(X, Y)$ is said to be strongly $\mathbf{L}^{2}$, if $S(\cdot) x \in \mathbf{L}^{2}(\mathbb{R} ; Y)$ for every $x \in X$. By the closed graph theorem one then has

$$
\|S\|_{2}:=\sup _{x \in X,\|x\| \leq 1}\|S(\cdot) x\|_{\mathbf{L}^{2}(\mathbb{R} ; Y)}<\infty .
$$

The mapping $S$ is said to be uniformly $\mathbf{L}^{2}$ if there exists a function $g \in \mathbf{L}^{2}(\mathbb{R})$ such that $g \geq 0$ and

$$
\|S(t) x\|_{Y} \leq g(t)\|x\|_{X} \quad(t \in \mathbb{R}, x \in X) .
$$

The function $g$ is said to be a scalar majorant of $S$. We will have occasion to use the following form of Young's inequality.

LEMMA 1.1 (Young's inequality). Let $X, Y$ be Banach spaces, let $S: \mathbb{R} \longrightarrow \mathcal{L}(X, Y)$ be strongly $\mathbf{L}^{2}$, and let $T: \mathbb{R} \longrightarrow \mathcal{L}(Y, Z)$ be uniformly $\mathbf{L}^{2}$ with scalar majorant $g$. Then the convolution $T * S$ defined by

$$
(T * S)(t) x:=\int_{\mathbb{R}} T(t-s) S(s) x d s \quad(x \in X, t \in \mathbb{R}),
$$

exists and satisfies $(T * S) \in \mathbf{C}_{\mathbf{0}}\left(\mathbb{R} ; \mathcal{L}_{S}(X, Z)\right)$ with

$$
\sup _{t \geq 0}\|(T * S)(t)\|_{\mathcal{L}(X, Y)} \leq\|g\|_{2}\|S\|_{2}
$$

One may choose $Y=Z$ and $T(s)=f(s) I, g(t)=|f(t)|$ in the lemma, so the estimate (1.3) becomes

$$
\sup _{t \geq 0}\|(f * S)(t)\|_{\mathcal{L}(X, Y)} \leq\|f\|_{2}\|S\|_{2}
$$

and this shows that with $S$ fixed the mapping

$$
(f \longmapsto f * S): \mathbf{L}^{2}(\mathbb{R}) \longrightarrow \mathbf{C}_{\mathbf{0}}(\mathbb{R} ; \mathcal{L}(X, Y)),
$$

is continuous. On the other hand, if we choose $X=\mathbb{C}$, then (1.3) shows that with fixed $T$ the mapping

$$
(f \longmapsto T * f): \mathbf{L}^{\mathbf{2}}(\mathbb{R} ; Y) \longrightarrow \mathbf{C}_{\mathbf{0}}(\mathbb{R} ; Z),
$$

is continuous.

For $N>0$ we denote by $D_{N}$ the Dirichlet kernel, that is

$$
D_{N}(t):=\frac{\sin (N t)}{\pi t} \quad(t \in \mathbb{R}) .
$$


Then, as is well known (or by a short computation),

$$
D_{N} * f=\frac{1}{2 \pi} \int_{-N}^{N} e^{i s t} \widehat{f}(s) d s
$$

where $f$ is integrable and $\widehat{f}=\mathcal{F} f$ denotes its Fourier transform. (Of course, the function $f$ may be vector- or operator-valued.) The following is an easy consequence of Plancherel's theorem.

LemMa 1.2. Let $f \in \mathbf{L}^{2}(\mathbb{R})$. Then $D_{N} * f \rightarrow f$ in $\mathbf{L}^{\mathbf{2}}(\mathbb{R})$ as $N \rightarrow \infty$.

\section{General Laplace transforms}

Let $X, Y$ be Banach spaces and let $S:[0, \infty) \longrightarrow \mathcal{L}(X, Y)$ be a strongly continuous mapping of finite exponential type $\omega_{0}(S)$. Note that

$$
\begin{aligned}
K_{N}(t) & :=\frac{1}{2 \pi i} \int_{\omega-i N}^{\omega+i N} e^{t z}(\mathcal{L} S)(z) d z \\
& =e^{\omega t} \frac{1}{2 \pi} \int_{-N}^{N} e^{i s t}(\mathcal{L} S)(\omega+i s) d s=e^{\omega t}\left(D_{N} * S_{\omega}\right)(t),
\end{aligned}
$$

whence $\left(K_{N}\right)_{\omega}=D_{N} * S_{\omega}$. If we replace $S$ by $a * S$ with a scalar function $a$ we arrive at our first result.

Proposition 2.1. Let $X, Y$ be Banach spaces, let $S:[0, \infty) \longrightarrow \mathcal{L}(X, Y)$ be strongly continuous, and let $a \in \mathbf{L}_{\mathrm{loc}}^{\mathbf{1}}[0, \infty)$ be a scalar function, both a and $S$ of finite exponential type. Then for every $\omega>\omega_{0}(S), \omega_{0}(a)$ one has

$$
\lim _{N \rightarrow \infty} \frac{1}{2 \pi i} \int_{\omega-i N}^{\omega+i N} e^{t z} \mathcal{L}(a * S)(z) d z=a * S,
$$

in $\mathcal{L}(X, Y)$, uniformly in t from compact subsets of $[0, \infty)$.

PROOF. Replace $S$ by $a * S$ in (2.1) to obtain

$$
e^{-\omega t} K_{N}(t)=D_{N} *(a * S)_{\omega}=D_{N} * a_{\omega} * S_{\omega},
$$

by (1.2). Now $D_{N} * a_{\omega} \rightarrow a_{\omega}$ in $\mathbf{L}^{2}(\mathbb{R})$ and hence, by Young's inequality, $D_{N} * a_{\omega} *$ $S_{\omega} \rightarrow a_{\omega} * S_{\omega}$ in $\mathcal{L}(X)$, uniformly in $t \geq 0$. Multiplying everything by $e^{\omega t}$ concludes the proof.

Proposition 2.1 does not quite cover [1, Theorem 2.3.4]; however, it will suffice for the applications we have in mind, and it is certainly more general than [3, Lemma 5], where the authors need $a \in \mathbf{C}^{\mathbf{1}}$ and assert only strong convergence and uniformity in $t$ from compact subsets of $(0, \infty)$. 
We would like to point out that we do not claim that Proposition 2.1 is new, although it might be (as we do not know of a reference). Our emphasis is on the idea of the proof, which can be put as follows. The complex inversion formula is simply the convergence of the partial inverse Fourier transforms. In a first step one establishes $\mathbf{L}^{2}$ convergence; then a convolution with another $\mathbf{L}^{2}$ term yields uniform convergence to something which, with some luck, is just a weighted form of what one is interested in.

In the following this idea is applied to the case of the complex inversion formula in its bare (that is, non-integral) form. The following observation will also be helpful.

Lemma 2.2. Let $X, Y$ be a Banach space, let $S:[0, \infty) \longrightarrow \mathcal{L}(X, Y)$ be strongly continuous of finite exponential type, and let $\omega>\omega_{0}(S)$. Then

$$
t\left(D_{N} * S_{\omega}\right) \sim\left(D_{N} *[t S(t)]_{\omega}\right)
$$

by which we mean that for each $x \in X$

$$
\lim _{N \rightarrow \infty}\left[t\left(D_{N} * S_{\omega}\right)-\left(D_{N} *[t S(t)]_{\omega}\right)\right] x=0,
$$

uniformly in $t \geq 0$.

PROOF. We perform integration by parts to obtain

$$
\frac{1}{2 \pi} \int_{-N}^{N} t e^{i s t} \widehat{S_{\omega}}(s) d s=\left.\frac{1}{2 \pi i} e^{i t s} \widehat{S_{\omega}}(s)\right|_{s=-N} ^{s=N}-\frac{1}{2 \pi i} \int_{-N}^{N} e^{i s t}{\widehat{S_{\omega}}}^{\prime}(s) d s,
$$

and clearly $\widehat{S}_{\omega}^{\prime}=-i \widehat{\left[t \widehat{S(t)}_{\omega}\right.}$. The statement now follows from the RiemannLebesgue lemma.

REMARK. The proof of [1, Theorem 2.3.4] relies on [1, Lemma 2.3.5] which states that

$$
\frac{1}{2 \pi i} \int_{\omega-i N}^{\omega+i N} \frac{e^{z t}}{z} e_{-z} d z \rightarrow \chi_{[0, t]} \quad(N \rightarrow \infty, t>0, \omega>0),
$$

in the Banach space $X=\mathbf{L}^{\mathbf{1}}[0, \infty)$ where $e_{-z}=e^{-z}$. This in fact follows from Proposition 2.1: let $S$ be the right shift semigroup on $X$ and $a=\mathbf{1}$. Then $(\mathbf{1} * S)(t)$ is convolution with $\chi_{[0, t]}$ and $(\mathcal{L} S)(z)$ is convolution with $e_{-z}$, as is easily seen. However, the $\mathcal{L}(X)$-norm of the operator 'convolution with $f$ ' equals the $\mathbf{L}^{\mathbf{1}}$-norm of $f$ (see [7] for a simple proof).

\section{Semigroups}

In this section we apply the results of the previous section to $C_{0}$ semigroups. Although it is a special case of the situation considered in Section 4, it is worthwhile to deal with the semigroup case first. We begin with the complex inversion formula in its integral form, see [5, Theorem III.5.14]. 
Theorem 3.1. Let A generate a bounded $C_{0}$ semigroup $(T(t))_{t \geq 0}$ on a Banach space $X$. Then for every $\epsilon>0$,

$$
\int_{0}^{t} T(s) d s=\lim _{N \rightarrow \infty} \frac{1}{2 \pi i} \int_{\epsilon-i N}^{\epsilon+i N} \frac{e^{z t}}{z} R(z, A) d z,
$$

in norm, the convergence being uniform in trom bounded intervals of $[0, \infty)$.

ProOf. This follows from Proposition 2.1 with $X=Y, S=T$ and $a=\mathbf{1}$.

Now we pass to the plain form of the inversion formula. To formulate it we need the notion of a UMD space, due to Burkholder. There are several equivalent definitions, one of which involves so-called $u$ nconditional martingale differences, but we will use a different characterization, due to Burkholder and Bourgain, involving the vectorvalued Hilbert transform (see [2] for more about UMD spaces). Given a function $m \in \mathbf{L}^{\infty}(\mathbb{R})$ one considers the operator $T_{m}$ defined by

$$
T_{m} f=\mathcal{F}^{-1}(m \widehat{f}),
$$

for $f$ from the Schwartz space $\mathcal{S}(\mathbb{R} ; X)$. (The symbol $\mathcal{F}^{-1}$ denotes the inverse Fourier transform.) The function $m$ is called a bounded $\mathbf{L}^{2}(\mathbb{R} ; X)$-Fourier multiplier if the operator $T_{m}$ extends to a bounded operator on $\mathbf{L}^{2}(\mathbb{R} ; X)$. If $m=\widehat{\mu}$ for some bounded measure $\mu \in \mathbf{M}(\mathbb{R})$, then $m$ is a bounded $\mathbf{L}^{2}(\mathbb{R} ; X)$-Fourier multiplier and $T_{m} f=\mu * f$. A Banach space $X$ is called a UMD space if the function

$$
h(t):=-i \operatorname{sgn}(t) \quad(t \in \mathbb{R}),
$$

is a bounded $\mathbf{L}^{2}(\mathbb{R} ; X)$-Fourier multiplier. The operator $\mathcal{H}:=T_{h}$ is called the Hilbert transform. It is known that there is a wealth of UMD spaces; for example, each Hilbert space is UMD (by Plancherel's theorem) and if $X$ is UMD and if $(\Omega, \Sigma, \mu)$ is a measure space, then $\mathbf{L}^{p}(\Omega, \Sigma, \mu ; X)$ is $\operatorname{UMD}$, for every $p \in(1, \infty)$. See [6, Appendix E] for more information and literature about UMD spaces.

Using little more than the definition of a UMD space, we can prove the vectorvalued analogue of Lemma 1.2.

Lemma 3.2. Let $X$ be a UMD space and $f \in \mathbf{L}^{\mathbf{2}}(\mathbb{R} ; X)$. Then $D_{N} * f \rightarrow f$ in $\mathbf{L}^{2}(\mathbb{R} ; X)$ as $N \rightarrow \infty$.

Proof. Consider the convolution operators $L_{N}:=\left(f \longmapsto D_{N} * f\right)$ on $\mathbf{L}^{\mathbf{2}}(\mathbb{R} ; X)$. By (1.4) one has $L_{N}(f)=D_{N} * f=\mathcal{F}^{-1}\left(\mathbf{1}_{[-N, N]} \widehat{f}\right)$, whence $L_{N}=T_{m_{N}}$ with $m_{M}:=\mathbf{1}_{[-N, N]}$. However,

$$
m_{N}=\mathbf{1}_{[-N, N]}=\frac{i}{2}(h(\cdot+N)-h(\cdot-N)) \quad(N>0) .
$$

Since $X$ is a UMD space, $h$ is a bounded $\mathbf{L}^{2}(\mathbb{R} ; X)$-Fourier multiplier. It is now easily seen that for each $r \in \mathbb{R}$ the shifted function $h(\cdot+r)$ is a bounded $\mathbf{L}^{\mathbf{2}}(\mathbb{R} ; X)$-Fourier multiplier as well, with same norm as $T_{h}=\mathcal{H}$. Hence, 


$$
\sup _{N}\left\|L_{N}\right\|_{\mathcal{L}\left(\mathbf{L}^{2}(\mathbb{R} ; X)\right)} \leq\|\mathcal{H}\|_{\mathcal{L}\left(\mathbf{L}^{2}(\mathbb{R} ; X)\right)}<\infty
$$

Since $\mathbf{L}^{\mathbf{2}}(\mathbb{R}) \otimes X$ is dense in $\mathbf{L}^{\mathbf{2}}(\mathbb{R} ; X)$ and $D_{N} * f \rightarrow f$ for scalar-valued $f$ by Lemma 1.2 , the statement follows.

Recall that for any $C_{0}$ semigroup $T$ on a Banach space $X$ one has

$$
(T * T)(t)=\int_{0}^{t} T(t-s) T(s) d s=t T(t) \quad(t \geq 0) .
$$

The definition of $K_{N}$ from (2.1) in this context reads

$$
K_{N}(t):=\frac{1}{2 \pi i} \int_{\omega-i N}^{\omega+i N} e^{t z} R(z, A) d z,
$$

where $A$ is the generator of $T$. Here is finally the result about strong convergence of the complex inversion formula.

THEOREM 3.3. Let $A$ be the generator of a $C_{0}$ semigroup $T$ on the Banach space $X$, let $\omega>\omega_{0}(T)$, and define $K_{N}$ by (3.3). Suppose that $X$ is a UMD space. Then for every $x \in X$

$$
t K_{N}(t) x \rightarrow t T(t) x \quad \text { as } N \rightarrow \infty,
$$

uniformly in t from bounded subintervals of $[0, \infty)$.

Proof. By (2.1) one has $K_{N}=e^{\omega t}\left(D_{N} * T_{\omega}\right)$. Now Lemma 2.2 and (3.2) yield

$$
e^{-\omega t} t K_{N}=t\left(D_{N} * T_{\omega}\right) \sim D_{N} *[t T(t)]_{\omega}=D_{N} * T_{\omega} * T_{\omega}=T_{\omega} *\left(D_{N} * T_{\omega}\right) .
$$

Fix $x \in X$. By Lemma 3.2, $D_{N} * T_{\omega} x \rightarrow T_{\omega} x$ in $\mathbf{L}^{2}(\mathbb{R} ; X)$ as $N \rightarrow \infty$. Young's inequality therefore yields $T_{\omega} *\left(D_{N} * T_{\omega} x\right) \rightarrow T_{\omega} * T_{\omega} x=[t T(t) x]_{\omega}$ uniformly in $t \geq 0$. Multiplying by $e^{\omega t}$ concludes the proof.

Applying Theorem 3.3 with $A=0$ and $X=\mathbb{C}$ yields the following.

Corollary 3.4. Let $\omega>0$. Then $\lim _{N \rightarrow \infty} t\left(D_{N} * \mathbf{1}_{\omega}\right)=t \mathbf{1}_{\omega}$ uniformly in $t \geq 0$.

Without $X$ being a UMD space, the theorem cannot be true, the canonical counterexample being the shift semigroup on $\mathbf{L}^{\mathbf{1}}(\mathbb{R})$; see [1, Example 3.12.3]. The classical result [1, Proposition 3.12.1, 5, Corollary III.5.15] is that one always has strong convergence if $x \in \mathcal{D}(A)$. We aim at showing that one actually has convergence in the norm of $\mathcal{L}\left(X, X_{-1}\right)$, where $X_{-1}$ is the first extrapolation space (see [5, Section II.5]).

THEOREM 3.5. Let $A$ be the generator of a $C_{0}$ semigroup $T$ on the Banach space $X$. Take $\omega>\omega_{0}(T)$ and define $K_{N}$ by (3.3). Let $\lambda \in \varrho(A)$ be arbitrary. Then

$$
\lim _{N \rightarrow \infty} t K_{N}(t) R(\lambda, A)=t T(t) R(\lambda, A),
$$

in norm, uniformly in t from bounded subintervals of $[0, \infty)$. 
Proof. By shifting the generator, we can assume that $\omega_{0}(T) \geq 0$, so $\omega>0$. We abbreviate $R:=R(\lambda, A), C:=A R(\lambda, A)$. The fundamental formula for semigroups reads $T-I=A(\mathbf{1} * T)$. Multiplying this by $R$ from the right yields

$$
T R=R+(\mathbf{1} * T) C .
$$

So

$$
e^{-\omega t} K_{N} R=D_{N} * T_{\omega} R=\left(D_{N} * \mathbf{1}_{\omega}\right) R+\left(D_{N} * \mathbf{1}_{\omega} * T_{\omega}\right) C .
$$

By the now well-known arguments, the second summand tends to $\mathbf{1}_{\omega} * T_{\omega} C=$ $[\mathbf{1} * T C]_{\omega}$ uniformly in $t \geq 0$. By Corollary 3.4 we know that $t\left(D_{N} * \mathbf{1}_{\omega}\right) \rightarrow t \mathbf{1}_{\omega}$ uniformly in $t \geq 0$. To sum up, we obtain

$$
\lim _{N \rightarrow \infty} t K_{N} R=e^{\omega t}\left(t \mathbf{1}_{\omega} R+t[\mathbf{1} * T]_{\omega} C\right)=t(R+(\mathbf{1} * T) C)=t T(t) R,
$$

in norm, and the convergence is uniform on bounded subintervals of $[0, \infty)$.

REMARK (Integrated semigroups). One may ask for analogues of Theorems 3.3 and 3.5 for integrated semigroups. Let $\alpha>0$, and suppose that $A$ generates an $\alpha$-times integrated semigroup $S$. Then $S$ and $A$ satisfy the equation

$$
S=\varphi_{\alpha}+A(\mathbf{1} * S)
$$

with $\varphi_{\alpha}(t):=t^{\alpha} / \Gamma(\alpha+1), t>0$. Following the lines of the proof of Theorem 3.5 one establishes that

$$
\lim _{N \rightarrow \infty} t K_{N}(t) R(\lambda, A)=t S(t) R(\lambda, A),
$$

in norm, uniformly in $t$ from bounded subintervals of $[0, \infty)$. (The argument is not completely analogous; to deal with the summand $t\left[D_{N} *\left(\varphi_{\alpha}\right)_{\omega}\right]$ one has to employ Lemma 2.2 and the identity $t \varphi_{\alpha}(t)=(\alpha+1) \varphi_{\alpha+1}=(\alpha+1)\left[\mathbf{1} * \varphi_{\alpha}\right]$. $)$

Apart from the trivial case where $A$ is a bounded operator, we do not know whether the analogue of Theorem 3.3 holds for integrated semigroups.

\section{Volterra equations}

The previous results on semigroups are only special cases of a more general theorem on (scalar-type) Volterra equations. In this case one is given a function $a \in \mathbf{L}_{\text {loc }}^{\mathbf{1}}[0, \infty)$ and one considers the abstract Volterra equation

$$
u=x+A(a * u) \quad(x \in X) .
$$

The well-posedness of this equation corresponds to the existence of a strongly continuous solution family $(S(t))_{t \geq 0}$ satisfying

$$
\int_{0}^{t} a(t-s) S(s) x d s \in \mathcal{D}(A) \text { and } \quad S(t) x-x=A \int_{0}^{t} a(t-s) S(s) x d s,
$$


for every $x \in X, t \geq 0$. In short notation, this means just

$$
S-I=A(a * S) .
$$

In case $a \equiv 1, S$ is a semigroup. It is convenient (and usual) to assume that $a$ and $S$ are of finite exponential type $\omega_{0} \geq 0$. In that case $S$ and $a$ are Laplace transformable and

$$
H(z):=\mathcal{L}(S)(z)=R(z, z(\mathcal{L} a)(z) A) \quad\left(\operatorname{Re} z>\omega_{0}\right) .
$$

As in the case of semigroups, one can ask under what conditions and in what sense the inversion of the Laplace transform converges to $S$. The definition of $K_{N}$ now reads

$$
K_{N}(t):=\frac{1}{2 \pi i} \int_{\omega-i N}^{\omega+i N} e^{t z} H(z) d z,
$$

where as usual $\omega>\omega_{0}$ is fixed. The following result is the exact generalization of the corresponding result for semigroups.

Theorem 4.1. Let $a, S, A, H, K_{N}$ as above, and let $\lambda \in \varrho(A)$ be arbitrary. Then

$$
\lim _{N \rightarrow \infty} t K_{N}(t) R(\lambda, A)=t S(t) R(\lambda, A),
$$

in norm, uniformly in t from bounded subintervals of $[0, \infty)$.

PROOF. By definition of a solution family, one has $S=I+A(a * S)$, so $S R=$ $R+(a * S) C$, where $R:=R(\lambda, A)$ and $C:=A R(\lambda, A)$. Therefore, the proof of Theorem 3.5 carries over almost literally.

The analogue of Theorem 3.3 is not so easy to obtain, and in general will not hold without additional assumptions on $a$. The assumptions we make are of a technical kind, chosen to make our proof work. However, they are weaker and easier to verify than those in [3].

THEOREM 4.2. Let $S$ and $a$ be as before. Suppose that there exists $b \in \mathbf{L}_{\text {loc }}^{\mathbf{1}}[0, \infty)$ such that the following holds:

(1) $(a * b)(t)=t a(t), t \geq 0$;

(2) $b_{\omega} \in \mathbf{L}^{2}(0, \infty)$

(3) $\quad\left(b^{\prime} * S\right)_{\omega}$ is uniformly $\mathbf{L}^{2}$.

(Here $b^{\prime}$ is the distributional derivative of $b$ on $\mathbb{R}$.) Define

$$
K_{N}(t):=\frac{1}{2 \pi} \int_{-N}^{N} e^{t(i s+\omega)} H(i s+\omega) d s .
$$

Then if $X$ is a UMD space, $\lim _{N \rightarrow \infty} t K_{N}(t) x=t S(t) x$ uniformly in $t$ from compact subsets of $[0, \infty)$, for each $x \in X$. 
Before we give the proof, which is more or less along the lines of the semigroup case, let us comment on the theorem. Condition (1) says that $\mathcal{L}(b)=-\mathcal{L}(a)^{\prime} / \mathcal{L}(a)$. The crucial point in applying the theorem is therefore to be able to recognize $\mathcal{L}(a)^{\prime} / \mathcal{L}(a)$ as a Laplace transform. The second is no essential condition, as we might always choose $\omega$ large enough. The third is more delicate, as it imposes regularity on $b$. We need that $b^{\prime} * S$ is meaningful, hence $b^{\prime}$ should be a Radon measure, that is $b \in \mathbf{B V}_{\text {loc }}[0, \infty)$. A feasible condition that implies (2) and (3) is that $b_{\omega_{0}}$ is bounded and $\left(b^{\prime}\right)_{\omega}$ is a bounded measure.

EXAmple 1. Let $\alpha=0$ or $\operatorname{Re} \alpha>0, a(t)=t^{\alpha}(t>0)$, and $\omega>0$. Then $b(t) \equiv \alpha+1$ clearly satisfies (1) and (2). Moreover, $b^{\prime}=(\alpha+1) \delta_{0}$ and so (3) is also satisfied. The case $\alpha=0$ recovers the semigroup case; the case $\alpha=1$ corresponds to $S$ being a cosine function.

Example 1 also appears in [3, p. 191]; however, in our case it is much easier to verify.

ProOF OF THEOREM 4.2. As in the semigroup case we apply Lemma 2.2 and obtain

$$
e^{-\omega t} t K_{N}=t\left(D_{N} * S_{\omega}\right) \sim D_{N} *[t S(t)]_{\omega},
$$

so we have to analyse $\mathcal{L}(t S(t))=-H^{\prime}$ further. Observe that $\mathbf{1} * b^{\prime}=(\mathbf{1} * b)^{\prime}=$ $\mathbf{1}^{\prime} * b=\delta_{0} * b=b$ and hence

$$
z(\mathcal{L} b)(z)=z \mathcal{L}\left(\mathbf{1} * b^{\prime}\right)(z)=z \frac{1}{z}\left(\mathcal{L} b^{\prime}\right)(z)=\left(\mathcal{L} b^{\prime}\right)(z) \quad\left(\operatorname{Re} z>\omega_{0}\right)
$$

Since $H(z)=R(z, z(\mathcal{L} a)(z) A)$, a little computation finally reveals that

$$
\begin{aligned}
H^{\prime}(z) & =\left(-\left(\frac{1}{z}+\frac{(\mathcal{L} a)^{\prime}(z)}{(\mathcal{L} a)(z)}\right)+z \frac{(\mathcal{L} a)^{\prime}(z)}{(\mathcal{L} a)(z)} H(z)\right) H(z) \\
& =(-(\mathcal{L} \mathbf{1})(z)+(\mathcal{L} b)(z)-z(\mathcal{L} b)(z) H(z)) H(z)
\end{aligned}
$$

see [3, Lemma 1]. (We used that $(\mathcal{L} b)(\mathcal{L} a)=\mathcal{L}(t a(t))=-(\mathcal{L} a)^{\prime}$.) Hence,

$$
\begin{aligned}
\mathcal{L}(t S(t)) & =-H^{\prime}=\left(\mathcal{L}(\mathbf{1}-b)+\mathcal{L}\left(b^{\prime} * S\right)\right)(\mathcal{L} S) \\
& =\mathcal{L}\left(\left[\mathbf{1}-b+\left(b^{\prime} * S\right)\right] * S\right),
\end{aligned}
$$

and by uniqueness of Laplace transforms, we obtain

$$
t S(t)=\left[\mathbf{1}-b+\left(b^{\prime} * S\right)\right] * S=C * S,
$$

with $C:=\left[\mathbf{1}-b+\left(b^{\prime} * S\right)\right]$. Thanks to hypotheses (2) and (3), $C_{\omega}$ is uniformly $\mathbf{L}^{\mathbf{2}}$, and so the same arguments as in the proof of Theorem 3.3 yield what we wanted to prove.

Let us point out that Theorem 4.2 is an improvement in comparison to [3, Theorem 1]. There it was required that: 
(1) $a(t)$ is 3-regular;

(2) $\mathcal{L} a=O\left(|z|^{-1}\right)$ for $|z|>1$;

(3) $\quad z(\mathcal{L} a)^{\prime}(z) /(\mathcal{L} a)(z)$ is locally analytic.

The authors do not specify the region of local analyticity, but from the proof it is clear that they mean locally analytic on $\mathbb{C}_{+}^{\infty}$. Since $(\mathcal{L} a)(z)$ does only exist for $\operatorname{Re} z>\omega_{0}$ the whole set of hypotheses seems a little strange; for example, in their definition of 3-regular (taken from [8, Definition 7.3]) one considers functions living on $\mathbb{C}_{+}$. However, with the help of our Theorem 4.2 we can relax hypotheses in the following way.

COROLLARY 4.3. Let $S$ be an exponentially bounded solution family for the scalartype Volterra equation (4.1), and let $\omega_{0}:=\max \left\{\omega_{0}(a), \omega_{0}(S)\right\}$. Suppose that the function $z(\mathcal{L} a)^{\prime} /(\mathcal{L} a)$ is locally analytic on $\left(\operatorname{Re} z>\omega_{0}\right) \cup\{\infty\}$. Then the hypotheses, and hence also the conclusion, of Theorem 4.2 hold true.

Proof. Let $F(z):=z(\mathcal{L} a)^{\prime} /(\mathcal{L} a)$. Then $F\left(z+\omega_{0}\right)$ is locally analytic on $\mathbb{C}_{+}^{\infty}$. So we may apply [8, Lemma 10.1] to conclude that there is a constant $c$ and a function $g \in \mathbf{L}^{\mathbf{1}}(0, \infty)$ such that $c+\mathcal{L} g=F\left(z+\omega_{0}\right)$, that is, $F=c+\mathcal{L} g-\omega_{0}$. Let $b:=\mathbf{1} * g_{-\omega_{0}}+c \mathbf{1}$. Then $b_{\omega_{0}}=\mathbf{1}_{\omega_{0}} * g+c \mathbf{1}_{\omega_{0}}$ is a bounded function and $\left(b^{\prime}\right)_{\omega}=$ $\left(g_{-\omega_{0}}\right)_{\omega}+c \delta_{0}=g_{\omega-\omega_{0}}+c \delta_{0}$ is a bounded measure. Hence, conditions (2) and (3) of Theorem 4.2 are satisfied. Furthermore, $\mathcal{L} b=z^{-1}\left(\mathcal{L} g_{-\omega_{0}}+c\right)=z^{-1} F(z)=$ $(\mathcal{L} a)^{\prime} /(\mathcal{L} a)$, and this is condition $(1)$.

\section{References}

[1] W. Arendt, C. J. K. Batty, M. Hieber and F. Neubrander, Vector-valued Laplace transforms and Cauchy problems, Monographs in Mathematics, 96 (Basel, Birkhäuser, 2001), pp. xi, 523.

[2] D. L. Burkholder, 'Martingales and singluar integrals in Banach spaces', in: Handbook of the geometry of Banach spaces, Vol. I (North-Holland, Amsterdam, 2001), pp. 233-269.

[3] I. Cioranescu and C. Lizama, 'On the inversion of the Laplace transform for resolvent families in UMD spaces', Arch. Math. (Basel) (2) 81 (2003), 182-192.

[4] A. Driouich and O. El-Mennaoui, 'On the inverse Laplace transform for $C_{0}$-semigroups in UMDspaces', Arch. Math. (Basel) (1) 72 (1999), 56-63.

[5] K.-J. Engel and R. Nagel, One-parameter semigroups for linear evolution equations, Graduate Texts in Mathematics, 194 (Springer, Berlin, 2000), pp. xxi, 586.

[6] M. Haase, The functional calculus for sectorial operators, Operator Theory: Advances and Applications, 169 (Birkhäuser-Verlag, Basel, 2006).

[7] - 'Semigroup theory via functional calculus', Preprint, 2006.

[8] J. Prüss, Evolutionary integral equations and applications, Monographs in Mathematics, 87 (Birkhäuser Verlag, Basel, 1993), pp. xxvi, 366.

\section{MARKUS HAASE, Delft Institute of Applied Analysis, TU Delft, PO Box 5031, 2600 GA Delft, The Netherlands e-mail: m.h.a.haase@tudelft.nl}

